\title{
Caracterización Geoestadística de una Probable Intrusión Magmática Activa, a Partir de la Sismicidad Reciente en el Estado de Hidalgo
}

\author{
José C. Escamilla-Casas ${ }^{\text {a,* }}$, Edgar M. Uribe-Alcántara ${ }^{a}$, Elvira Meneses-Meneses ${ }^{b}$, Silvia Montiel-Palmac, Vicente Rendón- \\ Hidalgo ${ }^{\mathrm{c}}$ \\ a Área Académica de Ciencias de la Tierra y Materiales, Universidad Autónoma del Estado de Hidalgo, Carr. Pachuca-Tulancingo km 4.5, Col. Carboneras, Mineral \\ de la Reforma, Hidalgo, C.P. 42184 \\ ${ }^{\mathrm{b}}$ Estudiante de Licenciatura en Ingeniería en Geología Ambiental, Área Académica de Ciencias de la Tierra y Materiales, Universidad Autónoma del Estado de \\ Hidalgo, Carr. Pachuca-Tulancingo km 4.5, Col. Carboneras, Mineral de la Reforma, Hidalgo, C.P. 42184 \\ ${ }^{c}$ Área Académica de Ingeniería y Arquitectura, Universidad Autónoma del Estado de Hidalgo, Carr. Pachuca-Tulancingo km 4.5, Col. Carboneras, Mineral de la \\ Reforma, Hidalgo, C.P. 42184
}

\begin{abstract}
Resumen
La sismicidad de baja intensidad y de profundidad somera es característica de la porción central del estado de Hidalgo. El análisis de estadística simple, Kriging ordinario y un mapa de densidad tipo Kernel, se han combinado para investigar la sismicidad en el centro de Hidalgo, durante 1990 a 2017. Los resultados muestran que la sismicidad somera se ha incrementado considerablemente en los últimos 4 años, independientemente de que se ha inferido un espesor de corteza continental de aproximadamente $45 \mathrm{~km}$, y sugieren que su origen se asocia con el emplazamiento de un cuerpo magmático semicircular activo. Adicionalmente, se propone un modelo tectónico dentro del contexto del sistema de subducción del sur de México y la evolución geológica del sector central de la Faja Volcánica Trans Mexicana.
\end{abstract}

Palabras Clave: Sismicidad, Estado de Hidalgo, Kriging, Kernel, Intrusión Magmática.

\section{Introducción}

En su mayor parte, México es un país catalogado como de alta sismicidad. Lo anterior es debido a que la configuración de su territorio se encuentra bajo la influencia de 5 placas tectónicas: Norteamericana, Pacífico, Cocos, Rivera y el Caribe. Al igual que en otras partes del planeta, la sismicidad más intensa se produce principalmente en los bordes de placas tectónicas que constituyen un sistema de subducción. Un sistema de subducción puede consistir en, al menos, una placa oceánica que se hunde en el manto terrestre por debajo del borde de una placa continental a lo largo de una trinchera. En México, tal sistema está constituido por las placas oceánicas de Rivera y Cocos que subducen, a lo largo de la Trinchera de Mesoamérica, a las placas continentales de Norteamérica y Caribe (Manea et al., 2013). Asimismo, la Faja Volcánica Trans Mexicana (FVTM) es un arco volcánico del Plioceno-Cuaternario, de aproximadamente $1000 \mathrm{~km}$ de longitud, que divide al territorio mexicano de este a oeste por su parte central, a lo largo de una franja entre los $19^{\circ}$ y $20^{\circ} \mathrm{N}$ (Figura 1). La FVTM está instaurada en el borde sur de la placa Norteamericana, se asocia genéticamente al sistema de subducción y actualmente presenta vulcanismo activo y sismicidad frecuente (Ferrari et al., 2012).

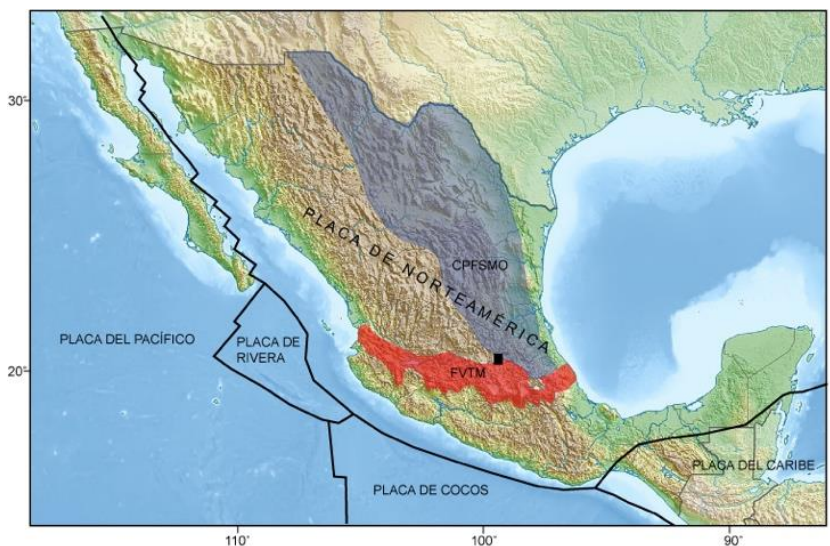

Figura 1: Mapa del territorio mexicano mostrando la ubicación de la zona de estudio (cuadro en negro), la Faja Volcánica Trans Mexicana (en rojo), el Cinturón de Pliegues y Fallas de la Sierra Madre Oriental (en azul oscuro), y la configuración de las placas tectónicas (Norteamericana, Pacífico, Rivera y Cocos) que constituyen al sistema en subducción en México.

El objetivo del presente trabajo es mostrar una interpretación novedosa basada en el análisis geoestadístico de la sismicidad somera en el estado de Hidalgo, mediante un modelo que relaciona la sismicidad actual con el emplazamiento de una probable intrusión magmática activa, en el marco tectónico de subducción del territorio Mexicano. Para esto, con base en la información topográfica de INEGI y los registros de sismicidad se generaron: un análisis de 
estadística simple, un modelo de elevación digital a partir de la información topográfica, un campo interpolado mediante Kriging de profundidad de hipocentros, y un mapa de densidad tipo Kernel ponderado por profundidad de hipocentros. El catálogo del Servicio Sismológico Nacional (SSN) presenta registros de la sismicidad en el estado de Hidalgo, comprendiendo un rango desde el primero de enero del año 1990 hasta el presente.

\section{Marco Geológico}

El área de estudio se localiza en la parte norte del Sector Central del FVTM, muy cercana al borde con el Cinturón de Pliegues y Fallas de la Sierra Madre Oriental, en el estado de Hidalgo (Figura 1).

El basamento de esta región está constituido por rocas sedimentarias del Mesozoico Superior y Terciario Inferior. Durante el pulso de deformación ocurrido durante el Eoceno (Fitz-Díaz et al., 20017), estas rocas fueron plegadas y traslapadas a lo largo de fallas inversas, conformando anticlinales y sinclinales con orientación preferencial norte-sur. Subsecuentemente, estas rocas fueron cubiertas discordantemente por tres secuencias de rocas volcánicas: la secuencia inferior consiste en riolitas, tobas, y flujos de andesita y basalto del Mioceno; la secuencia intermedia que se considera la parte basal de la FVTM con rocas del Plioceno al Mioceno y la tercera secuencia que la constituyen rocas calco-alcalinas del Plioceno-Cuaternario (Campos Enríquez et al., 2000).

En el área estudiada, además del pulso de deformación del Eoceno, ocurrieron eventos de deformación subsecuentes. Durante el Eoceno y el Oligoceno Temprano ocurrió un evento distensivo responsable de la formación de depresiones tectónicas. Durante el Mioceno y el Plioceno, fases subsecuentes de deformación ocurrieron generando fallas regionales con orientación preferencial Este-Oeste, con componentes de desplazamiento lateral izquierdo (Pasquaré et al., 1987).

Asimismo, en esta región se aloja la estructura geológica mayor denominada graben Mezquital (Sutter et al., 1995a y 1995b), el cual se caracteriza por una sismicidad moderada y somera (Sutter et al., 1996; Campos-Enríquez et al., 2000). Adicionalmente, se ubica la Caldera Mezquital, que corresponde a una estructura semicircular de $20 \mathrm{~km}$ de diámetro (Hernández Avelino, 2008).

\subsection{Sismicidad}

El Sector Central de la FVTM se considera sísmicamente activo. Sin embargo, la zona de estudio se caracteriza por una sismicidad de magnitud baja y profundidad somera. En trabajos previos, se han registrado eventos sísmicos relevantes ocurridos en el siglo pasado en el estado de Hidalgo (Tabla 1).

Tabla 1: Eventos sísmicos relevantes en Hidalgo

\begin{tabular}{cccccc}
\hline Fecha & $\begin{array}{c}\text { Hora } \\
\text { (hh:mm:ss) }\end{array}$ & $\begin{array}{c}\text { Lat. } \\
\mathrm{N}\end{array}$ & $\begin{array}{c}\text { Lon. } \\
\text { W }\end{array}$ & $\begin{array}{c}\text { Prof. } \\
\text { km }\end{array}$ & $\mathrm{M}_{\mathrm{c}}$ \\
\hline $10-14-48$ & $00: 19: 03$ & 20.12 & 99.12 & - & $4.5^{1}$ \\
$02-08-49$ & $17: 25: 35$ & 20.12 & 99.12 & - & $4.5^{1}$ \\
$03-11-50$ & $00: 42: 51$ & 22.35 & 98.97 & - & $5.02^{1}$ \\
$08-15-56$ & $22: 52: 27$ & 20.12 & 99.12 & - & $4.0^{1}$ \\
$03-25-76$ & $23: 05: 07$ & 20.58 & 99.02 & 15.3 & $5.3^{3}$
\end{tabular}

$\begin{array}{cccccc}04-05-76 & 10: 49: 12 & 20.46 & 99.09 & 10.4 & 4.2^{2} \\ 10-04-76 & 06: 59: 15 & 20.48 & 99.15 & 9 & 5.3^{5} \\ 02-06-81 & 18: 11: 49 & 20.01 & 99.03 & 6.2 & 3.4^{2} \\ 01-27-87 & 16: 33: 29 & 20.31 & 99.21 & 15 & 4.1^{3} \\ 06-02-88 & 12: 02: 31 & 20.29 & 99.05 & 6 & 2.7^{4}\end{array}$

${ }^{1}$ Figueroa, 1970; ${ }^{2}$ Bravo et al., 1988; ${ }^{3}$ Servicio Sismológico Nacional; ${ }^{4}$ Campos-Enríquez et al., 2000; ${ }^{5}$ Suárez y Ponce, 1986.

\section{Materiales y métodos}

En el presente estudio, se contemplaron los registros de sismicidad en el estado de Hidalgo proporcionados por el catálogo del SSN. Los sismos ocurridos se registraron mediante dos redes sísmicas que opera el SSN: la red sísmica de banda ancha nacional y la red sísmica del Valle de México.

Para el análisis que se presenta, se construyó una base de datos con la totalidad de los registros disponibles en el catálogo del SSN, comprendidos entre el 1 de enero de 1990 hasta el 31 de diciembre de 2017, considerando 334 registros de hipocentros en el estado de Hidalgo (Figura 2). Posteriormente, de acuerdo con la configuración presentada por Ferrari et al. (2012), en la que se determina un espesor de la corteza continental en la región de estudio entre 45 y 50 km, se generó una segunda base de datos en la que se excluyeron los hipocentros con una profundidad mayor a $32 \mathrm{~km}$. Lo anterior se hizo para estudiar la sismicidad en la parte superior de la corteza continental, excluyendo así a la zona cercana a la subducción. Los hipocentros así considerados están distribuidos entre un rango de profundidades de 1 a $32 \mathrm{~km}$ y entre los municipios de: Actopan, Ajacuba, Alfajayucan, Atitalaquia, Cardonal, Chilcuautla, El Arenal, Francisco I. Madero, Ixmiquilpan, Mixquiahuala de Juárez, Progreso de Obregón, San Agustín Tlaxiaca, San Salvador, Santiago de Anaya, Tetepango, Tezontepec de Aldama, Tlauhelilpan y Tlaxcoapan. Los campos que definen a ambas bases de datos son: fecha, hora, latitud, longitud, profundidad del hipocentro y magnitud del sismo.

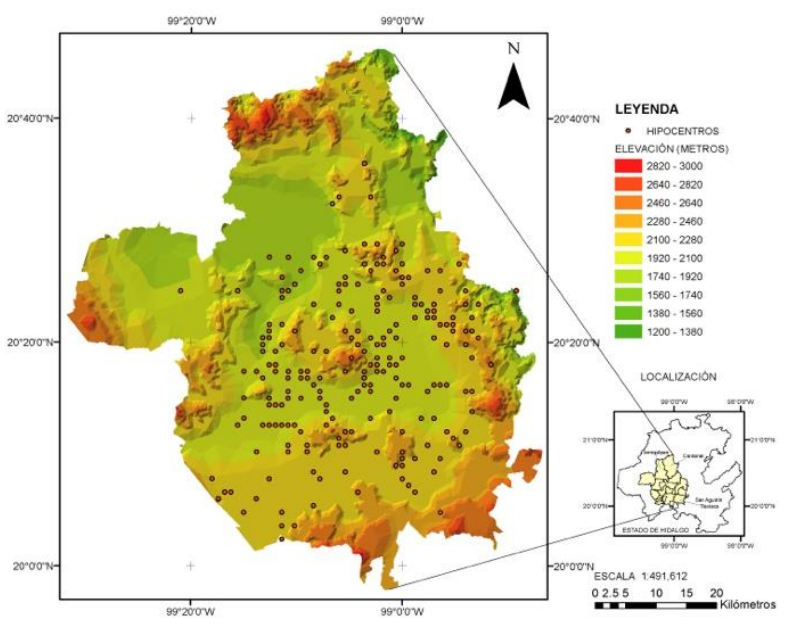

Figura 2: Modelo digital de elevación mostrando la ubicación de los hipocentros considerados en este estudio. En el inserto se muestra el contorno del estado de Hidalgo con los municipios en donde han ocurrido los sismos. 
Para la elaboración de los análisis Kriging ordinario y distribución de densidad Kernel se utilizó el programa computacional ArcMap. De la misma forma, se utilizó la misma versión de ArcMAp para manipular y referir geográficamente los datos sísmicos y topográficos, además de realizar el modelo digital de elevación. El programa Microsoft Excel v. 16.11.1 se utilizó para elaborar la estadística simple.

La base topográfica utilizada corresponde a las hojas INEGI F14D71, F14C88, F14V70, F14D81, F14C69 у F14C78; proyectadas en sistema de coordenadas UTM, elipsoide WGS84, zona14 norte.

Para generar el campo interpolado de la profundidad de los hipocentros, se eligió el método de interpolación Kriging (Giraldo, et al., 2011), dado que es un método exacto y proporciona una evaluación de la incertidumbre de los valores interpolados (Figura 4). Finalmente, se elaboró un mapa de distribución de densidades Kernel ponderado por profundidad de hipocentros (Figura 9). Con este método es factible investigar la densidad de hipocentros por unidad de área en la región, pero ponderada por la profundidad de hipocentros (Rudemo, 1982). Se decidió realizar ambos análisis porque Kriging nos permite asumir que es posible asociar la profundidad de los hipocentros con un límite estructural geológico. Mientras que, por otra parte, el análisis de densidad nos permite identificar aquellos lugares donde se concentran mayores cantidades de hipocentros. Como la densidad está ponderada por la profundidad, también nos permite identificar aquellos lugares donde los hipocentros son más profundos.

\section{Resultados}

Los resultados que se obtuvieron a partir de la estadística simple aplicada a la base de datos con todos los registros de sismos en el estado de Hidalgo demuestran que la sismicidad ha aumentado sensiblemente entre los años 2014 a 2017. Es decir, de los 334 sismos ocurridos entre el 1 de enero de 1999 y el 31 de diciembre de 2017, 212 sismos $(63.47 \%$ ) ocurrieron durante ese periodo (Figura 3).

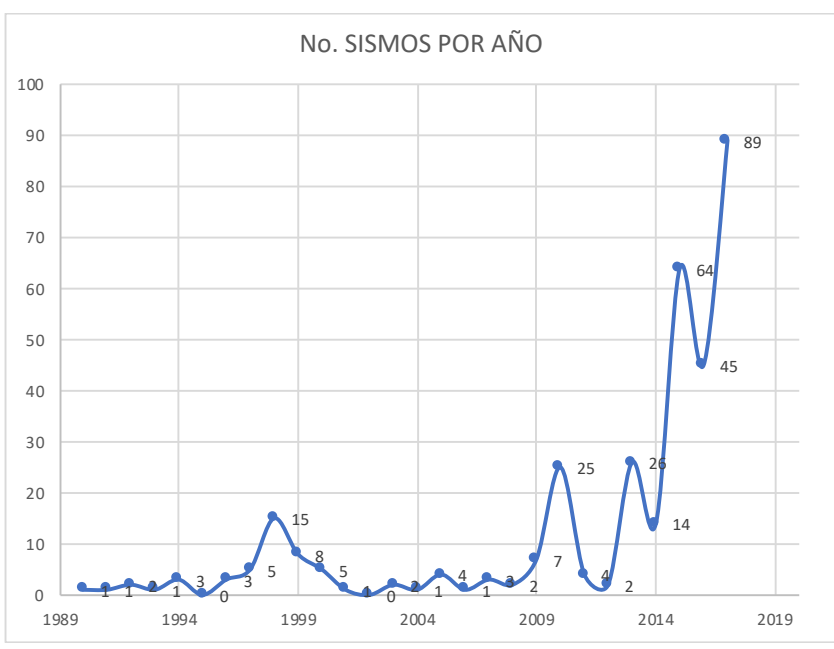

Figura 3: Gráfica que representa el número de sismos ocurridos por año en el estado de Hidalgo. Se observa que la sismicidad más intensa se presentó durante los años 2014 a 2017.
Previo a la interpolación con Kriging, se analizaron los histogramas de frecuencias de los hipocentros. Fue posible observar que la variable tiene un fuerte sesgo a la izquierda (Figura 4). Consecuentemente, se aplicó una transformación de tipo logarítmica, que permitió lograr una distribución más cercana a la gaussiana (Figura 5), que es la distribución recomendada para aplicar el método Kriging. También se efectuó un análisis de tendencia. Sin embargo, no se detectó una tendencia significativa. Por lo cual, se aplicó un análisis Kriging tipo ordinario con transformación logarítmica.

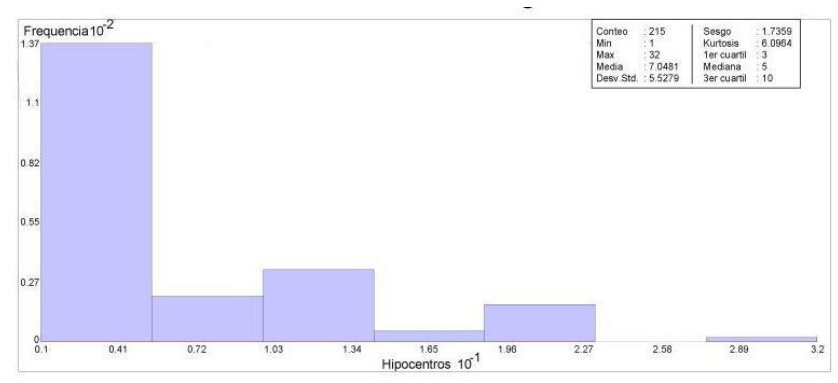

Figura 4: Histograma sin ninguna transformación con un sesgo muy pronunciado y una distribución muy lejana a la gaussiana.

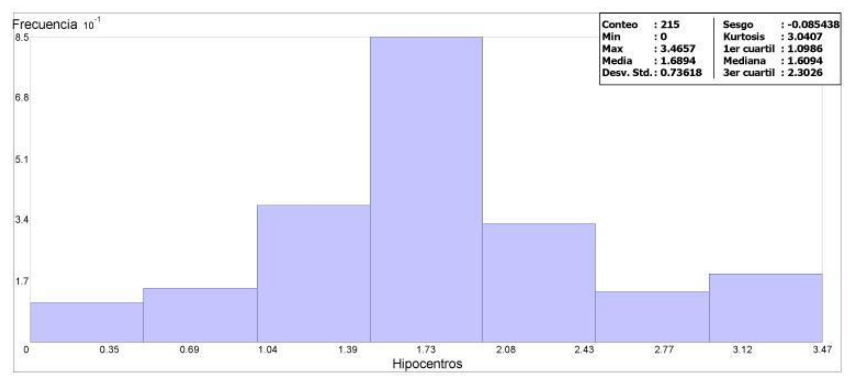

Figura 5: Histograma resultante de la transformación logarítmica. La distribución de los eventos es más cercana a la normal, aunque prevalece un ligero sesgo.

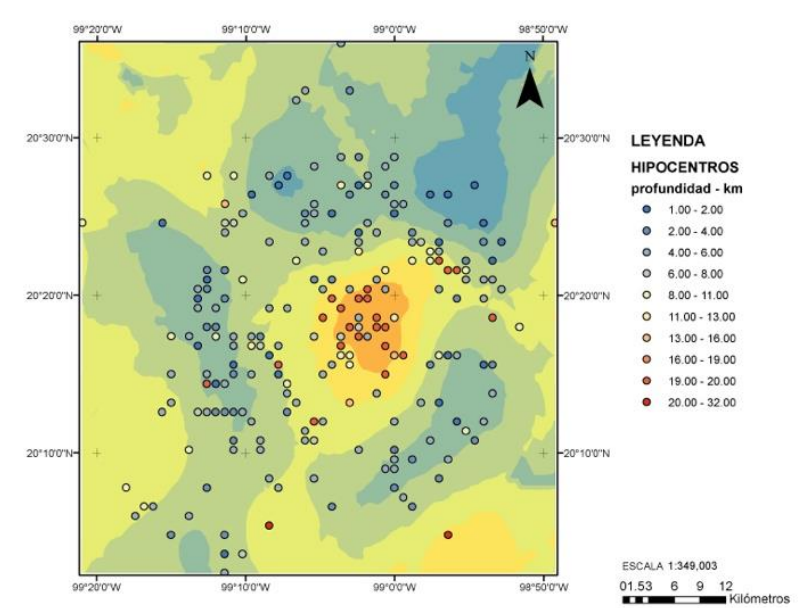

Figura 6: Figura mostrando el campo interpolado de los datos de profundidad de los hipocentros. Es notable la distribución que tiene ya que define una distribución semicircular.

En la Figura 6, se muestra el resultado del campo interpolado para representar una superficie estimada a partir de las 
profundidades de los hipocentros. La distribución de las profundidades en el campo interpolado describe valores máximos definiendo un cúmulo a profundidades que oscilan entre los 16 y $32 \mathrm{~km}$.

Por otro lado, los valores mínimos de profundidad (1-6 km) describen una configuración semicircular casi concéntrica con el cúmulo de los valores máximos. De igual manera, los valores de intermedios también se distribuyen de manera similar con respecto al cúmulo de profundidades mayores.

El mapa de densidad tipo Kernel ponderado por profundidad de hipocentros (Figura 7) muestra una configuración concéntrica. En el mapa se observa un par de cúmulos de valores máximos y que los valores intermedios y mínimos se distribuyen concéntricamente a su alrededor. Adicionalmente, la distribución circular que se muestra tanto en campo interpolado resultado del análisis Kriging es congruente con el mapa Kernel.

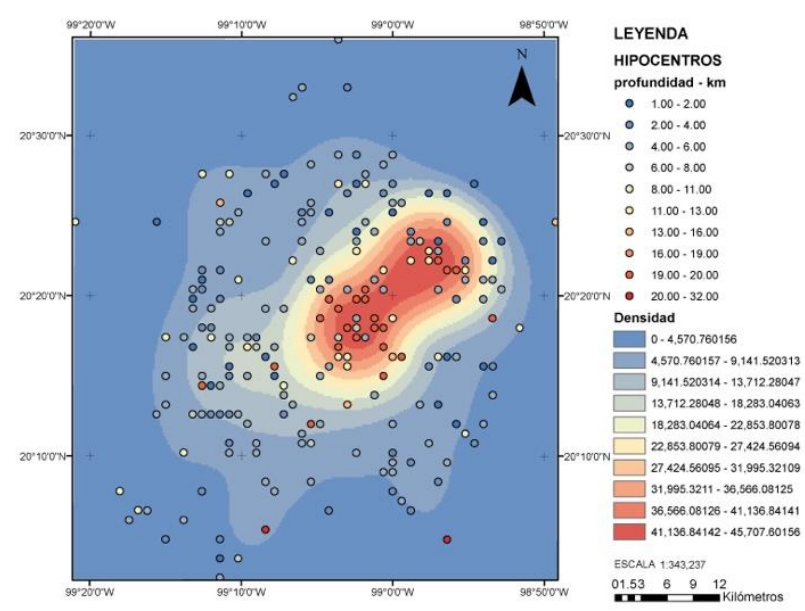

Figura 7: Distribución de los valores de densidad correspondiente al número de eventos ponderado por profundidad por unidad de área.

\section{Discusión}

En este estudio, se ha investigado la distribución de la sismicidad en el estado de Hidalgo bajo el contexto tectónico regional generalmente aceptado. Las observaciones que se derivan del desarrollo del presente estudio son que, en el sector central del estado de Hidalgo, la sismicidad que se presenta se define como de magnitud baja y de profundidad cortical somera. Sin embargo, en los últimos 4 años, la ocurrencia de eventos sísmicos se ha incrementado considerablemente. Por otro lado, se observa que la distribución espacial de los hipocentros describe una configuración semicircular y que la mayor densidad de estos se concentra en un punto central.

Derivado de lo anterior, se propone un modelo geológico que muy probablemente explica el origen de la sismicidad en Hidalgo (Figura 8). El modelo considera que un cuerpo magmático es derivado del proceso de subducción y generado por la fusión de las rocas de la placa continental y/o oceánica. Es probable que ese cuerpo magmático, por mecanismos de boyancia y fracturamiento, siga un camino ascendente hacia la parte superficial de la corteza. La distribución de la densidad de los hipocentros sugiere que el cuerpo magmático tiene forma irregular formando altos y bajos en profundidad, sin embargo, unidos a un cuerpo principal o fuente. La actividad constante en el proceso de subducción implica que el cuerpo magmático esté sujeto a movimiento ascendente. Por último, el incremento en la frecuencia de los sismos y con hipocentros someros, sugiere que el cuerpo magmático podría estar ubicado a poca profundidad.

No se ha encontrado documentación de este fenómeno en la literatura científica. Es prácticamente seguro que las autoridades y comunidades desconocen su ocurrencia. El incremento exponencial de la actividad sísmica sugiere que el fenómeno continuará acentuándose y, eventualmente, puede resultar en una erupción. Quizá la medida más recomendable, por el momento, sería la creación y operación de un sistema de monitoreo que permita seguir cercanamente la evolución del fenómeno. Este sistema de monitoreo debería considerar no solo la actividad sísmica, sino instrumentación adicional para el seguimiento cercano de la evolución del fenómeno. Una vez que se cuente con más información, y si se confirma que existe un riesgo para la comunidad, se considera importante acercarse a autoridades y comunidades para informarles de este fenómeno en desarrollo. En consecuencia, podrán tomar medidas precautorias oportunas.

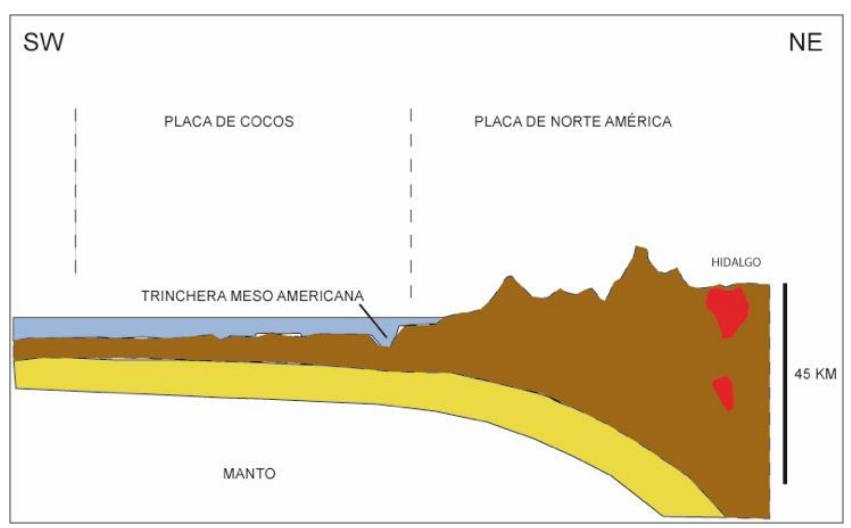

Figura 8: Modelo tectónico propuesto mostrando esquemáticamente la posición de las placas Norteamericana y Cocos, además de las ubicaciones de la Trinchera Meso Americana y el estado de Hidalgo. Los objetos en rojo indican la ubicación probable del cuerpo magmático con respecto al espesor de la corteza continental.

\section{English Summary}

Geostatistical Characterization of a Probable Active Magmatic Intrusion, Based on Recent Seismicity in the State of Hidalgo
Abstract
Typically, low intensity and shallow seismicity occurs in the central portion of the State of Hidalgo. Simple statistics, ordinary Kriging analysis, and a Kernel density map were combined in order to investigate the seismicity of central Hidalgo, between 1999 and 2017. Findings show that the shallow seismicity has increased in the past four years, in spite 
of an inferred continental crust thickness of ca. $45 \mathrm{~km}$, and strongly suggest the emplacement of an active, semicircular, magmatic body. Finally, a tectonic model is proposed in the context of the subduction system of southern Mexico and the geologic evolution of the central sector of the Trans Mexican Volcanic Belt, in Hidalgo.

\section{Keywords:}

Seismicity, Hidalgo, Kriging, Kernel map, Trans Mexican Volcanic Belt, subduction

\section{Agradecimientos}

Este trabajo ha sido realizado durante el desarrollo de los proyectos de investigación: "Caracterización en un Espacio Bidimensional de Fallas y Fracturas en la Sierra de Pachuca" y del proyecto "Desarrollo de una metodología para la Creación de mapas de riesgo estatales por procesos de Remoción de Masa con base en Sistemas de Información Geográfica".

\section{Referencias}

Bravo, H., Ponce, M.L., Suárez, R.G., Zúñiga, R., 1988, Potencial sísmico de México. Estudios sobre sismicidad en el Valle de México: México, D. F., Departamento del Distrito Federal, 45p.

Campos-Enríquez, J.O., Rodríguez, M., Delgado-Rodríguez, O., 2000, Contribution to the tectonics of the northern portion of the central sector of the Trans-Mexican Volcanic Belt. En: Delgado-Granados, H., Aguirre-Díaz, G.J., Stock, J. M., (Ed.), Cenozoic Tectonics and
Volcanism of Mexico. Geological Society of America, Special Paper 334, Boulder, Colorado, E.U.A., p.223-235.

Ferrari, L., Orozco-Esquivel, T., Manea, V., Manea, M., 2012. The dynamic history of the Trans-Mexican Volcanic Belt and the Mexico subduction Zone. Tectonophysics, 522-523, 122-149.

Fitz-Díaz, E., Lawton, T.F., Juárez-Arriaga, E., Chávez-Cabello, G., 2017 The Cretaceous-PAleogene Mexican orogen: Structure, basin development, magmatism and tectonics. Earth-Science Reviews, http://dx.doi.org/10.1016/j.earscirev.2017.03.002

Giraldo, R., Delicado, P., Mateu, J., 2011. Ordinary Kriging for FunctionValued Spatial Data. Environmental and Ecological Statistics, 18,3,411-426.

Hernández Avelino, I., 2008. Caracterización Geológica y Petrológica de la Estructura Circular Mezquital (Estado de Hidalgo) y su Posible Riesgo Geológico, Tesis Maestría. Instituto Politécnico Nacional, ESIA-Ticomán, División de Estudios de Posgrado, México D.F., 105 pp.

Manea, V.C., Manea, V., Ferrari, L., 2013. A geodynamical perspective on the subduction of Cocos and Rivera plates beneath Mexico and Central America. Tectonophysics 609, 56-81.

Rudeno, M., 1982. Empirical Choice of Histograms and Kernel Density Estimators. Scandinavian Journal of Statistics, 9,2,65-78.

Sutter, M., Quintero-Legorreta, O., López-Martínez, M., Aguirre-Díaz, G. Farrar, E., 1995a. The Acambay Graben: active intraarc extensión in the Trans-Mexican Volcanic Belt, Mexico. Tectonics 15, 1245-1262.

Sutter, M., Martínez, M.C., López-Martínez, M., Ferrar, E., 1995b. The Aljibes half graben - active extension at the boundary between the TMVB and the Basin and Range Province, Mex. Geological Society of America Bulletin 107, 627-641.

Sutter, M., Carrillo Martínez, M., Quintero-Legorreta, O., 1996. Macroscopic study of shallow earthquakes in the central and Eastern parts of the trans-mexican volcanic belt, Mexico. Bulletin of Seismological Society of America 86, 1952-1963.

Suárez, G., Ponce., L., 1986, Intraplate seismicity and crustal deformation in central Mexico [abs.]. Eos (Transactions, American Geophysical Union) 67,4 